\title{
Accuracy limit of modern ephemerides imposed by the uncertainties in asteroid masses
}

\author{
E. M. Standish ${ }^{1}$ and A. Fienga ${ }^{1,2}$ \\ 1 Jet Propulsion Laboratory, California Institute of Technology, JPL 301-150, Pasadena, CA 91109, USA \\ 2 Institut de Mécanique céleste et de calculs des éphémérides, 77 Av. Denfert-Rochereau, 75014 Paris, France
}

Received 19 November 2001 / Accepted 20 December 2001

\begin{abstract}
Accuracy limits in the ephemerides of the four inner planets, imposed by uncertainties in the masses of the asteroids, are investigated and illustrated. We consider present-day knowledge of the asteroid masses (determined by the IRAS survey, direct dynamical determinations, ground-based photometry, occultations, etc.), and we model the distribution of those masses. This distribution is then used in a Monte Carlo study, repeatedly adjusting the ephemerides to fit the observational data, each time using a different, but equally-likely, set of asteroid masses. The differences in the resulting ephemerides are shown. If the full inherent weighting of the highly accurate ranging data is used, stretching over more than two decades, the orbits become distorted in right ascension and declination - as much as 5 kilometers or more. If the ranging is de-weighted to a level equivalent to the other two coordinates (1-2 mas, determined by VLBI), then a reasonable ephemeris results, showing uncertainties of 2-3 kilometers. It is also possible to produce an ephemeris which will extrapolate a year or so into the future at the sub-kilometer level (as is often required for spacecraft navigation). This can be done by fully-weighting only the recent observational data. However, the ephemeris farther from the fitting interval is seen to deteriorate rapidly.
\end{abstract}

Key words. astrometry - celestial mechanics - ephemerides - solar system: minor planets - planets and satelites: general

\section{Introduction}

Modern ephemerides of the major bodies in the solar system represent one of the best-known dynamical systems in the history of mankind. The system is virtually free from friction, dust, and magnetic fields; it has remained undisturbed by outside forces; it has been measured for a long period of time with some of the most accurate measurements ever made; and, the equations of motion governing the major bodies are relatively simple, yet they do involve some features which are basic to physics.

The ephemerides are not perfectly known, however. For the major bodies (sun, the Moon, and planets), there are perturbations, arising from literally hundreds of asteroids, which are significant but which can not be modeled with sufficient accuracy. These perturbations can reach a number of kilometers in the case of Mars. In comparison, modern ephemerides are adjusted to electronic ranging measurements which are typically accurate to 10 meters or even less. Therefore, in order to calculate the motions

Send offprint requests to: A. Fienga,

e-mail: fienga@bdl.fr at a level comparable to the observations, the modeling of the asteroid perturbations would need to be accurate at the $1 \%$ level or less - far more accurate than present knowledge.

While the orbits of the asteroids are known with sufficient accuracy for the present purpose, most of their masses are not. As will be seen, for many asteroids, the estimated masses could well be in error by a factor of 2 or even more. As a consequence, the motions of the planets, especially Mars, are known and predictable with only limited accuracy.

In this paper we consider the inner planet system the sun, the Moon, and 4 innermost planets - since their ephemerides are tightly coupled due to the accurate ranging measurements to which they are fit. We concentrate on Mars and the Earth since they are most affected by the asteroid perturbations and since it is the observations and ephemeris of Mars which are most important in establishing the inertial mean motions of the inner planets (Williams 1984; Williams \& Standish 1989). We present an assessment of the uncertainties introduced into the ephemerides by the uncertainties in the masses of the 


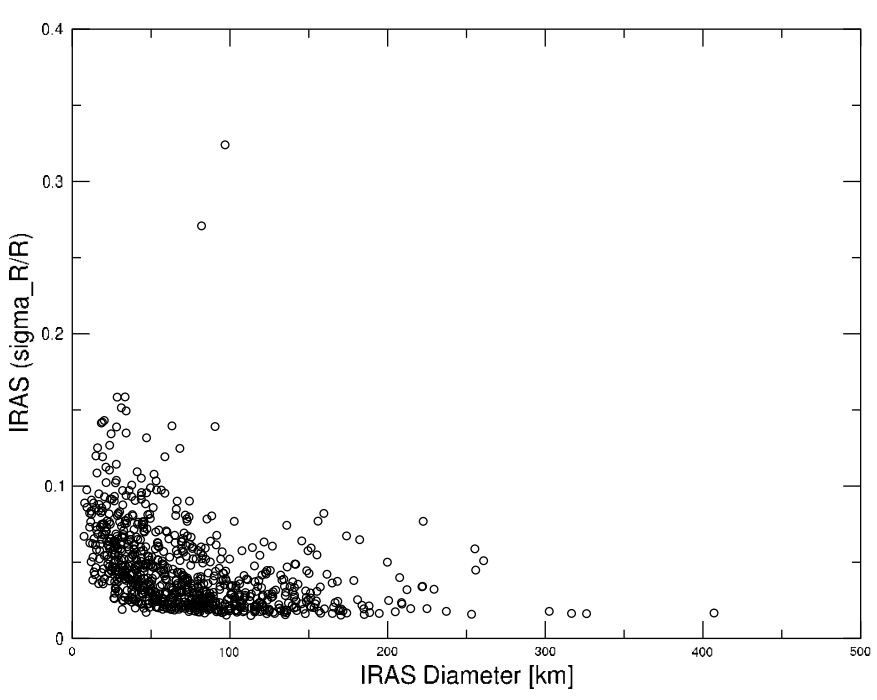

Fig. 1. IRAS 1 sigma distribution of errors. This distribution will be the basis of the Monte Carlo simulations.

asteroids. A distribution is chosen for the uncertainties in the adopted masses according to each asteroid's diameter and taxonomic class, and sets of masses are selected randomly from within that distribution. These sets of masses are then used for Monte Carlo simulations to integrate the effect upon the orbits of the Earth and Mars. Subsequent ephemeris adjustments are made with different weighting schemes, and the differences in the resultant ephemerides are studied.

\section{The types of ephemeris uncertainties for the inner planet system}

The uncertainties of the inner planet ephemerides may be roughly categorized into three main groups:

1. the relative angles and distances between the bodies,

2. the orientation of the system as a whole onto an external reference frame (now, the ICRF), and

3 . the mean motions of the bodies with respect to inertial space.

It is relatively easy to visualize the fact that relative positions are determined by ranging measurements over an interval in question, as long as the geometry of the system changes sufficiently during that time. It is also quite easy to visualize the orientation of the system being determined by ICRF-based VLBI measurements of a spacecraft orbiting one of the system's bodies (assuming the position of the spacecraft with respect to the planet is sufficiently well known). Less obvious is the fact that accurate ranging also determines the mean motions of the system's members with respect to inertial space (the dynamics would be different in a rotating system). See, e.g., Williams \& Standish (1989).

It is primarily the third of these types of uncertainties that is affected by the presence of the asteroids: the accumulating effect over time of the poorly-modeled asteroid forces, due to the uncertainties in the asteroids' masses.

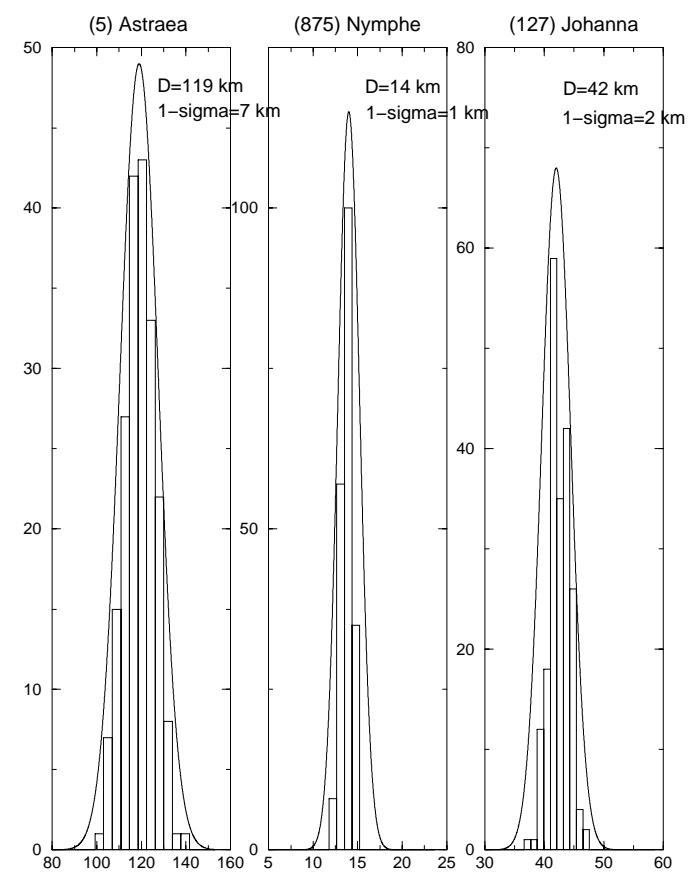

Fig. 2. Histograms of 200 simulations for the diameters of asteroids (5) Astraea $(119 \pm 7 \mathrm{~km}),(875)$ Nymphe $(14 \pm 1 \mathrm{~km})$ and (127) Johanna $(42 \pm 2) \mathrm{km}$.

\section{Asteroid masses and the modeling of their perturbations in present-day planetary ephemerides}

There are a few asteroids for which mass determinations have been made directly. For some others, either occultations of stars or radar echo measurements have provided estimations of the asteroid's diameter. For most of the other relatively large asteroids, diameter estimations have been made indirectly using the observations from IRAS (Infra-Red Astronomical Satellite). For the remaining larger asteroids, existing ground-based magnitude measurements are used with assumptions about the albedos.

For the creation process of the ephemerides (Standish 1998), Ceres, Pallas, and Vesta, the "Big 3", are handled separately. During the integration of the ephemerides, their positions are taken from a pre-integrated file and their forces upon the Moon and all of the planets are computed and applied in real time. For the ephemerides EPM2000 (Pitjeva 2001), the orbits of the "Big 3" were integrated simultaneously with those of the planets and the Moon.

For about 300 other asteroids, selected because of their non-negligible effect upon the orbits of the Earth, the Moon, and Mars, the estimated diameter is used to calculate the volume. These asteroids are also assigned to one of the three general taxonomic classes: C, S, or M (carbonaceous, silicate, or metallic). An adopted density for each class is used with the volumes in order to give an estimated mass. For each taxonomic class, the forces upon the Earth, the Moon, and Mars are summed for each 


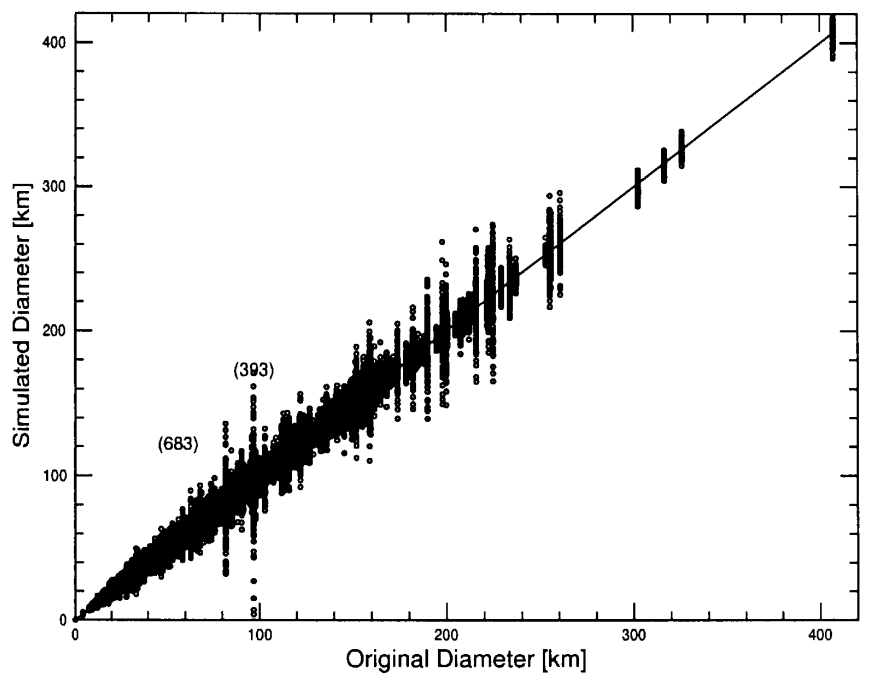

Fig. 3. New simulated diameters versus the original diameter values (IRAS, occultations, radar diameters). The biggest dispersions are produced by IRAS values. For example, (683) Lanzia is $(82 \pm 22) \mathrm{km}$ and (393) Lampetia $(97 \pm 31) \mathrm{km}$.

designated point in time and stored on a file. This file is subsequently interpolated during the integration and the forces are applied to the Earth, the Moon, and Mars.

During the fitting of the ephemerides, it is possible to solve for adjustments to the masses of each of the Big 3 and to the densities of each of the three taxonomic classes.

The errors in the asteroid masses come from, in the case of the Big 3, the direct estimations of their masses. In the case of the others, there are uncertainties in the diameter estimations, in the densities of the three classes, and also in the somewhat vague assignment of each asteroid to one of the three classes.

\section{Assessment of ephemeris errors from asteroid mass uncertainties}

If a different (but equally likely) set of asteroid masses were used in the ephemeris adjustment process, what difference would it make? The answer to such a question should give an idea of how much error is introduced into the ephemerides by the uncertainties in the asteroid masses. Thus, we choose an alternate set of asteroid masses, integrate the new ephemerides, and re-adjust them, weighted according to the existing observations. We then compare the newly, re-adjusted ephemerides with the original ephemerides. We describe in 4.1 a simplified equation of motion used for the integration, in 4.2 and 4.3 the two different ways of choosing the alternate mass sets, in 4.4 the integration and the re-adjustment, paying attention to the weighting scheme, and in 4.5 the results the differences in the ephemerides.

\subsection{Equation of motion}

For the integration, we use a common differential method. Only the difference, $\delta \boldsymbol{r}$, between an initial system and its

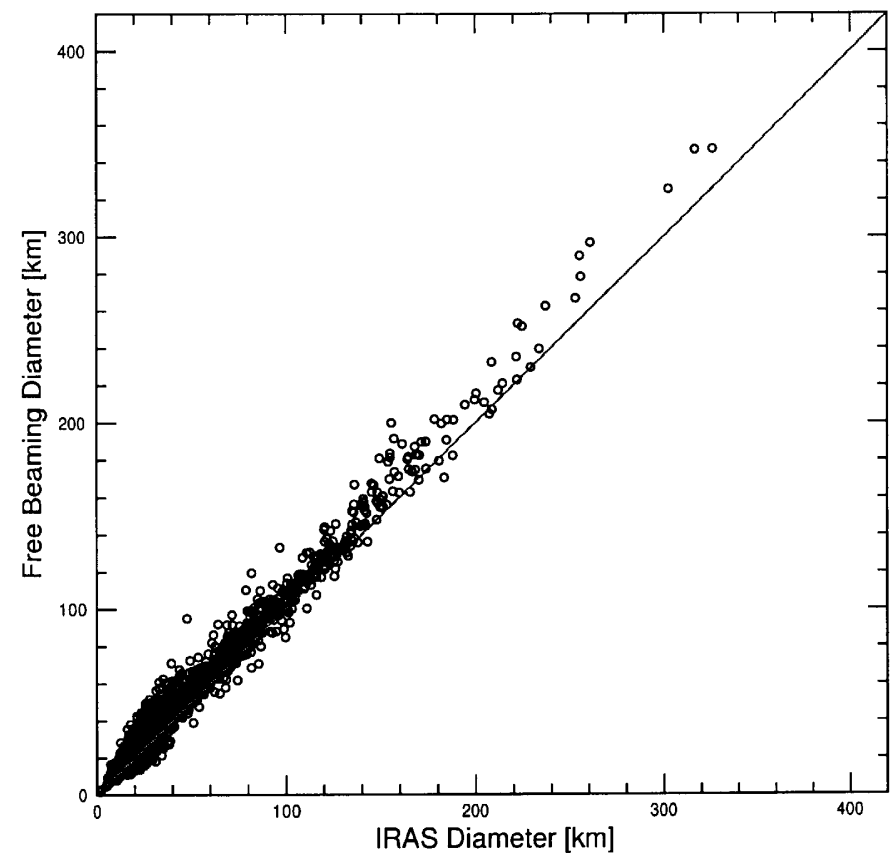

Fig. 4. 1884 values of asteroid diameters estimated by IRAS and by the Free Beaming method (Hasegawa 1999).

counterpart with an added set of $n$ perturbing bodies is integrated:

$\delta \ddot{\boldsymbol{r}}=-G\left(m_{\odot}+m_{\mathrm{o}}\right)\left[\frac{\delta \boldsymbol{r}}{r^{3}}-3 \frac{\boldsymbol{r}(\boldsymbol{r} \cdot \delta \boldsymbol{r})}{r^{5}}\right]+\sum_{i=1}^{n} G m_{i}\left[\frac{\boldsymbol{\rho}_{\boldsymbol{i}}{ }^{\prime}}{\rho_{i}^{\prime 3}}-\frac{\boldsymbol{r}_{i}}{r_{i}^{3}}\right],(1)$

where $m_{\odot}$ is the mass of the central body, $m_{\mathrm{o}}$ is the mass of the perturbed body, $\boldsymbol{r}$ is the position of the perturbed body with respect to the central body, $\boldsymbol{\rho}_{\boldsymbol{i}}{ }^{\prime}=\boldsymbol{r}_{i}-\boldsymbol{r}-\delta \boldsymbol{r}$, and where $m_{i}$ and $\boldsymbol{r}_{i}$ are the mass and the position of the ith perturber. Each perturber may be a totally new body $\left(m_{i}\right)$ or an additional mass added to one of the existing bodies $\left(\delta m_{i}\right.$ instead of $\left.m_{i}\right)$.

\subsection{Choosing the alternative sets of masses, $\delta M$}

One way to select an alternate mass set, is to simulate a new one. To create a new set of masses, we consider various simulated values of the mass uncertainties.

The randomly chosen masses have to reflect our relatively good knowledge of the sizes of big asteroids and our relatively poor estimations of diameters smaller than a few tens of kilometers. The IRAS catalog gives values for both the diameter and the corresponding $1 \sigma$ uncertainty. The distribution of the ratios $\sigma_{R}$ over $R$, plotted in Fig. 1, gives a good idea of the status of our knowledge of asteroid diameters and of the present limitation in such estimations. Occultation and radar estimations of diameters are also used in this work, corresponding to $2.6 \%$ of the whole sample. For each observed asteroid, we simulate a Gaussian distribution centered on the diameter value, $\mu_{\mathrm{r}}$ and with a sigma from the IRAS, occultation and radar values, $\sigma_{\mathrm{r}}$. At each run, a random selection is made according to the distribution. If $z$ is a such random 
Table 1. Actual observations used in modern ephemeris for fitting of the Mars and Earth orbits. The weights are computed considering $n$, the number of observations, the time-coverage, and $\sigma$, the a priori accuracy of the measurements.

\begin{tabular}{ccccccc}
\hline & & & & & Post Fit 1 & Post Fit 2 \\
& Period & Observation Type & Planet & $n$ & $\sigma$ & $\sigma$ \\
\hline$(\alpha, \delta)$ & $1990-94$ & Magellan VLBI & Venus & 18 & $3 \mathrm{~km}$ & $3 \mathrm{~km}$ \\
& $2001-3$ & MGS + Odyssey VLBI & Mars & 34 & $1 \mathrm{~km}$ & $1 \mathrm{~km}$ \\
& & & & & \\
$\rho$ & $1976-81$ & Viking Lander ranging & Mars & 1282 & $10 \mathrm{~m}$ & $1 \mathrm{~km}$ \\
& 1997 & Pathfinder ranging & Mars & 90 & $10 \mathrm{~m}$ & $1 \mathrm{~km}$ \\
& $1998-2001$ & MGS ranging & Mars & 200 & $10 \mathrm{~m}$ & $1 \mathrm{~km}$ \\
\hline
\end{tabular}

variable, then its probabilty density function is (Fishman 1995):

$f(z)=\frac{1}{\sqrt{2 \pi \sigma_{\mathrm{r}}^{2}}} \exp ^{-\frac{\left(z-\mu_{\mathrm{r}}\right)^{2}}{2 \sigma_{\mathrm{r}}^{2}}}$,

Examples of 200 simulations are plotted for 3 asteroids in Fig. 2.

For the taxonomic densities, a simulation of the errors is also performed for each object. For these computations, a Gaussian distribution is assumed, centered on the density values and with a $1 \sigma$ equivalent to $20 \%$. The taxonomic classification is extracted from the Small Bodies Data Archives (SBDA, 2001).

For the object which have a spectral signature near the $\mathrm{C}, \mathrm{S}$ and $\mathrm{M}$ classes, the values of the bulk densities are those obtained by Standish (2000). For the objects near the $\mathrm{V}, \mathrm{B}$ and $\mathrm{G}$ classes, the bulk densities are deduced from the computation of the masses of Ceres, Pallas and Vesta (Standish 2000) and from a given value of diameter (usually the diameter obtained by occultation). The selected values of the $\mathrm{V}, \mathrm{B}$ and $\mathrm{G}$ classes are respectively $(3.44 \pm 0.12),(2.71 \pm 0.11)$ and $(2.12 \pm 0.04)$. The choise of the value of the bulk density is not really critical because the random simulation of the value of the taxonomic densities is such as it could simulate the error in the estimation of a bulk density for a given class but also the error in taxonomic classification.

For each run, randomly chosen diameter and density values are simulated for a list of 880 asteroids (Ceres, Pallas and Vesta excluded) which includes the 300 asteroids used in the planetary ephemerides, plus 580 others, whose radii are greater than 5 kilometers.

In Fig. 3, are plotted the new randomly simulated diameters against the initial set (IRAS, occultations, radar diameters). New $\delta M_{i}$ are established for each object (represented by the $m_{i}$ in Eq. (1)), and used to compute new orbits of Mars and of the Earth using Eq. (1).

\subsection{An existing $\delta M$ set: IRAS-FBM}

With the new release of the IRAS catalog (Tedesco 1992), 1884 asteroids have had new estimations of their diameters and albedos. With these estimations, a lot of discussion considering the reduction process of the IRAS survey has arisen. Several attempts to improve the IRAS results were made by Harris \& Harris (1997), Lupishko (1998) and Hasegawa (1999), using different types of thermal models. Differences in the results obtained by each of these works tend to indicate the external accuracy of the IRAS data. Figure 4 represents the estimations by IRAS and by the Free Beaming Model, FBM (Hasegawa). The differences between IRAS and the FBM show a significant bias, especially for small objects: the IRAS diameters seem to be under-estimated compared to the FBM values. This is an example of how the value of the asteroid diameters could be affected by observational bias or bias produced by the choice of the reduction model. Added to a $20 \%$ error on the bulk density estimations, we obtain the (IRAS-FBM) $\delta M$. This $\delta M$ represents a possible amount of mass error introduced by the IRAS diameters and by the approximate determination of the bulk densities.

\subsection{Integration and re-adjustement}

We simulate full ephemeris re-adjustments necessitated by the different sets of asteroid masses, $\delta M$ :

1. Given a set of masses, $\delta M$, Eq. (1) is integrated over a 40-year interval, giving $\delta \boldsymbol{r}\left(=\boldsymbol{r}_{\mathrm{O}}-\boldsymbol{r}_{\mathrm{C}}\right)$, the difference between the new ephemeris and the base ephemeris;

2. Residuals, $\alpha_{\mathrm{O}}-\alpha_{\mathrm{C}}, \delta_{\mathrm{O}}-\delta_{\mathrm{C}}$, and $\rho_{\mathrm{O}}-\rho_{\mathrm{C}}$ (geocentric $\mathrm{RA}$, Dec, and range) are computed;

3. The residuals are weighted in order to properly reflect the quantity, accuracy, and time-span of the existing actual observations (Table 1);

4. Adjustments to the initial conditions are determined in order to best-fit the weighted residuals;

5. The subsequent changes to the ephemeris are shown by linearized new residuals.

In practice, the residuals were formed at uniformly-spaced points in time. In the least squares adjustments, however, these were properly weighted in order to reflect the observations given in Table 1.

As seen in Table 1, two fits were actually formed for each set of $\delta M$. The first used the full weighting for all 
observations; for the second, the ranging observations were severely down-weighted. The reason for this is discussed below.

\subsection{Results}

Figure 5 presents ten examples of the runs using different sets of asteroids masses. For each example, the prefit differences (pseudo-residuals) are given in right ascension, declination, and range, along with two sets of post-fit residuals. We also used the $\delta M$ set, IRAS-FBM, shown in Fig. 5 and denoted by the number 0 . The runs numbered 1-9 are from the first 9 of the 100 randomly produced sets of the $\delta M$.

For the pre-fit plots, the residuals in each coordinate are seen to grow from the initial values of 0 at the starting epoch of the integrations, 1969 Jun. 28 (JED 2440400.5). They often reach 2 or more kilometers in value after a few decades.

In each case, there is a drift in right ascension ( longitude) of the new ephemeris, either ahead or behind the base ephemeris. The spikes are geometrical, occurring at Mars' oppositions. For the most part, the declination and range residuals simply reflect the perturbations in right ascension.

Two sets of post-fit residuals, also shown in Fig. 5, result from two different weighting schemes used in the orbital adjustments. For the first set, Post Fit 1, the adjustments were made in all three coordinates (RA, Dec, and range), and the weights reflected the number, accuracy, and time-spans of the actual observations which are presently being used or will soon be used in modern-day ephemeris adjustments (see Table 1). For this set, the overwhelming accuracy of the ranging data dominates the system to such an extent that the post-fit residuals in RA and Dec are actually much larger than in the pre-fit cases. As will be discussed, this is a direct result of trying to fit sets of data too accurately when there are mis-modeled forces (in this case, wrong values for the asteroid masses). For the second set of post-fit residuals, Post Fit 2, the ranging data were severely down-weighted so that the accuracy of the ranging observations was comparable to that of the angular (VLBI) measurements. In these sets, the residuals become very small during the time where the angular measurements exist (1990-2003) and are correspondingly larger for the earlier decades.

In all, 100 cases similar to those in Fig. 5 were run. All 100 are plotted on top of each other in Fig. 6 , in order to emphasize the major features:

1. the general size of the ephemeris differences caused by the alternate sets of asteroid masses;

2 . the de-fitting of the angular residuals when the accurate ranging measurements stretch over an extended period of time (long enough so that the errors from the mis-modeling can accumulate);
3. the ability to produce accurate $(<1 \mathrm{~km})$ ephemerides, but only over a relatively short interval containing accurate observations, and

4. the deterioration of the ephemeris away from the fitting interval (due to the uncertainties in the asteroid masses).

\subsection{Discussion}

Figure 6 can be interpreted as the covariance of the Earth-Mars ephemeris. Since there were 100 simulations all plotted in Fig. 6, one can consider the outer envelope to be nearly the size of a curve representing the $3 \sigma$ uncertainties.

The covariance represented by Fig. 6 is only an approximate estimate. In this case, there have been a number of estimations of the uncertainties involved in the asteroid masses: the diameter uncertainties enter into the volume as the third power; the density of each taxonomic class is unknown and could very well vary between the individual members of the class; and the assignment of each asteroid to a particular taxonomic class is often tentative at best.

The sets of $\delta M$ represent unmodeled forces, introducing pseudo-random noise into the dynamical system which accumulates over time. For this reason, and as shown in Fig. 6, it is not possible to connect the Earth-Mars ephemeris over two decades with an accuracy comparable to that of the measurements themselves. The attempts to do so, shown in the first set of post-fit residuals in Fig. 6, tie the range down during 1976-82 (Viking) and 19972003 (Pathfinder and MGS). However, the orbit distorts noticeably in right ascension and declination, because the accuracy of the angular measurements is not comparable to that of the ranging.

One can, on the other hand, tie down the ephemeris with high accuracy, but only during a short interval of time. The second sets of post-fit residuals show a tight fit in all three dimensions at the time of the angular data, but the residuals remain small for only a few years around that time.

The angular data is sufficient to determine the EarthMars range at the $1 \mathrm{~km}$ level, since the range is tightly coupled to the planets' longitude difference through the equation, $\rho^{2}=r_{1}^{2}+r_{2}^{2}+r_{1} r_{2} \cos \left(\lambda_{2}-\lambda_{1}\right)$. I.e., we could have eliminated the range data altogether in the second set of post-fit residuals, and we would have still seen an uncertainty in range of a size comparable to that in right ascension and declination. Alternatively, we could have included the ranging data at full strength over just the interval of Pathfinder and MGS; that dimension would then show an uncertainty to match the data, but only for that short span in time. We have also performed other experiments in which we introduce a positive bias, such as in the FBM diameters for the FBM-IRAS $\delta M$ set (set Number 0 in Fig. 5). In all cases, the drift in right ascension is positive. A negative bias, correspondingly, produces a negative drift. The implications are that a positive bias 
Prefit

Right ascension
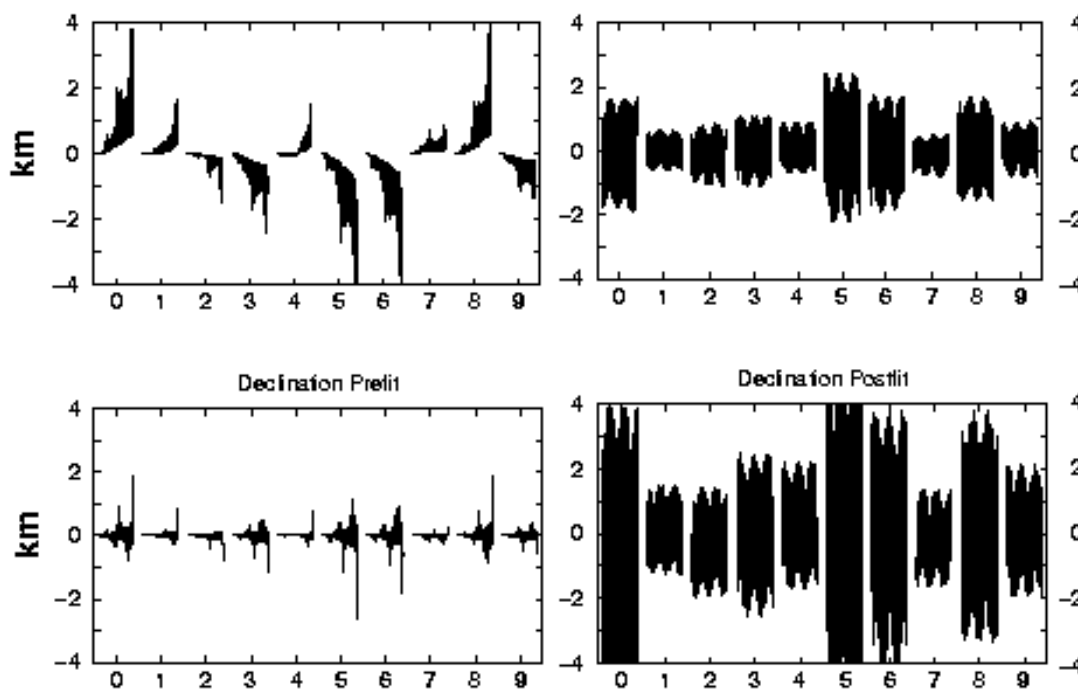

Right ascension
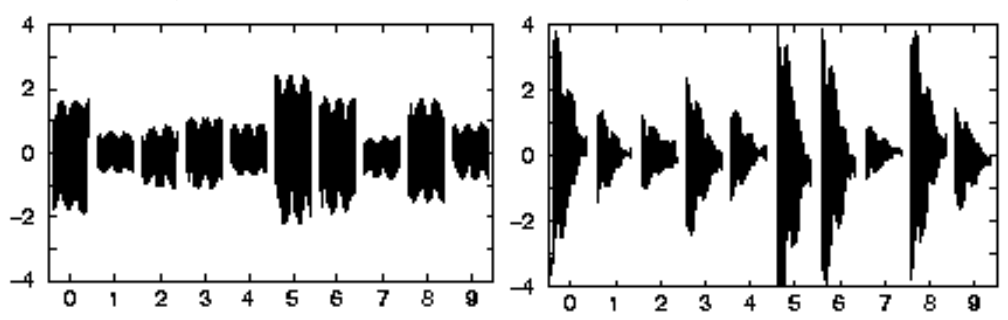

Post Fit 2

Right ascension
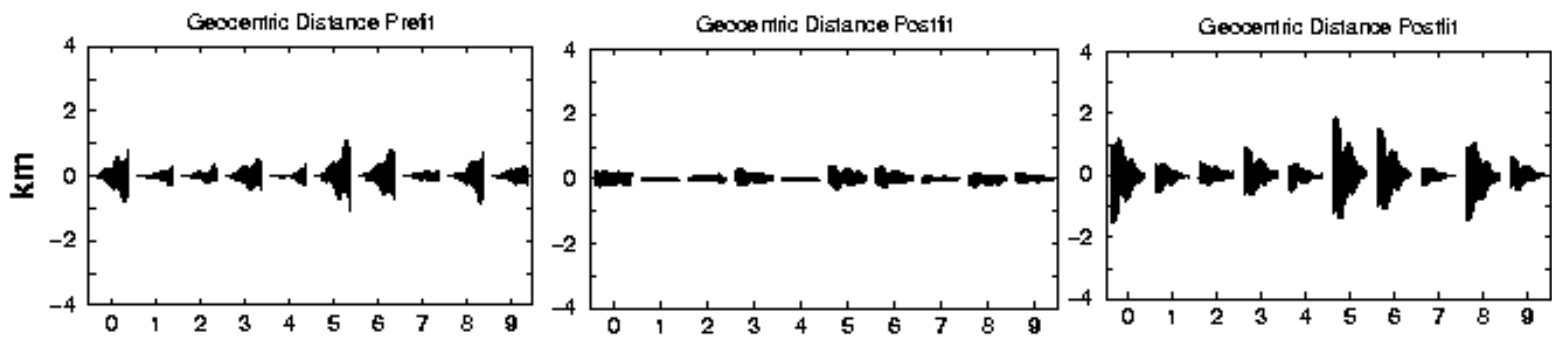

Fig. 5. Examples of ephemeris adjustements using 10 differents sets of $\delta M$. Set Number 0 is from FBM-IRAS; $1-9$ are randomly produced according to a specific distribution function. Two differents weighting schemes are used for the adjustements (see text).

in the asteroids diameters will produce a positive drift in the right ascension of Mars. This will be compensated for by a negative bias in the estimated asteroids densities used for computing the masses.

Can the modeling be improved for better ephemeris creation? The following points are relevant:

1. Certainly, the best known set of asteroid masses should be carefully selected for use in creating the ephemerides;

2. The possibility of solving in the ephemeris solutions for a significant number of individual asteroid masses is not yet a realistic one; longer stretches of highly accurate observations are needed;

3. Any independent asteroid mass determinations would be of help. Unfortunately, spacecraft determinations are too few and dynamical determinations (deducing one asteroid's mass from its perturbations upon another) have had rather poor success so far in most cases, as noted by Krasinsky et al. (2001);

4. There is the possibility of grouping the asteroids, not according to taxonomic class, but rather according to the periods of their perturbations upon the EarthMars ephemeris. If the spread of such frequencies within each group would be small enough, then the amplitude and phase of each group's perturbation would remain fairly constant over a few years, allowing one to solve for the phases and amplitudes and to then make short-term predicitions into the future;

5. Krasinsky et al. (2001) and Bange (1998) have introduced a massive ring to account for the many small asteroids. Krasinsky et al. have estimated this ring for asteroids beyond the 300 that have been considered so far. They find a significant amount of mass for this ring - a mass comparable to the mass of Ceres.

\section{Conclusion}

The uncertainties of the masses of the asteroids in the solar system introduce uncertainties into the Earth-Mars ephemeris which can amount to several kilometers over the course of a decade or so. This is greater than the accuracy of the present-day VLBI measurements and far greater than any spacecraft-ranging measurements.

The Earth-Mars ephemeris uncertainties over time are strongly dependent upon the choice of the weighting scheme used in fitting the observations. If full weight is given to the ranging observations, the ephemeris will tend to distort in the other dimensions in order to accommodate the highly accurate ranging. On the other hand, there 
Post Fit 1
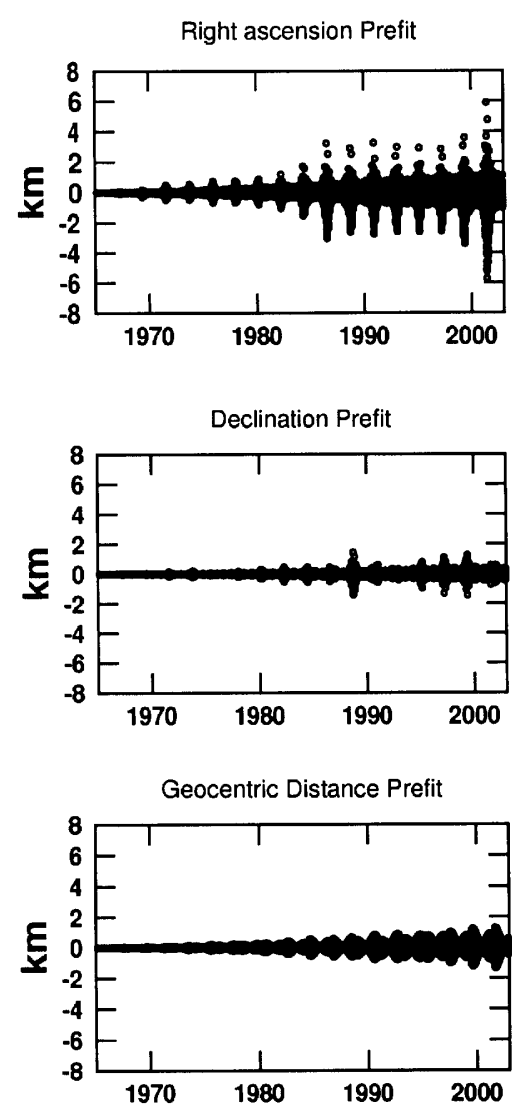

Right ascension Postfit

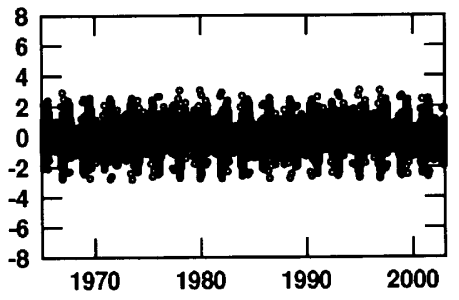

Declination Postfit

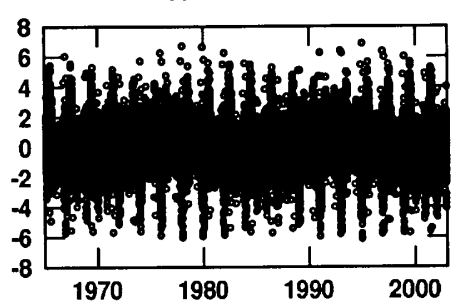

Geocentric Distance Postfit

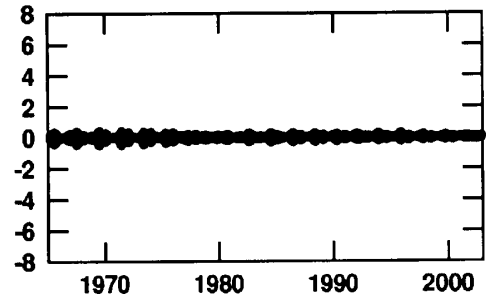

Post Fit 2

Right ascension Postfit

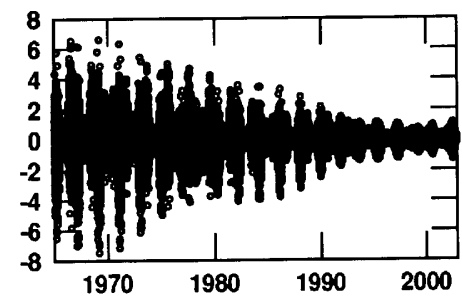

Declination Postfit

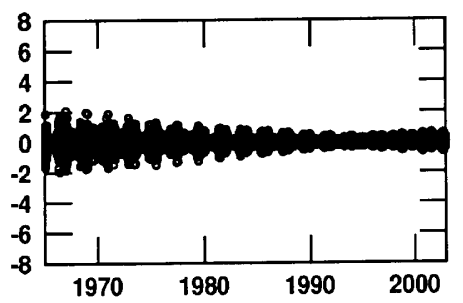

Geocentric Distance Postit

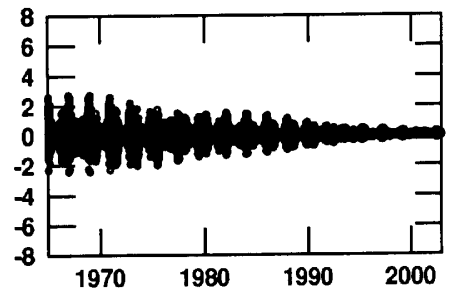

Fig. 6. Superimposition of ephemeris adjustements for all 100 sets of $\delta M$. The outer envelope may be considered as an approximation to a 3 sigma covariance. The weighting schemes and the shapes of the plots are discussed in the text.

is valid information in the ranging data; this implies the need for continuing attempts to better represent the asteroid perturbations.

For accurate, short-term ephemerides, where extrapolation into the near future is important, such as that needed for spacecraft navigation, it is best to confine the full weighting to only the recent data. Current VLBI and ranging serve to establish the ephemeris at the present time; the effect of the older observations, such as the Viking ranging, must be lessened by down-weighting those observations, or by introducing bias parameters, or by some other method.

Acknowledgements. The work described in this paper was carried out at the Jet Propulsion Laboratory, California Institute of Technology, under contract with the National Aeronautics and Space Administration and thanks to the Lavoisier French grant.

\section{References}

Bange, J.-F. 1998, Ph.D. Thesis, Observatoire de Paris Cellino, A., Zappala, V., \& Farinella, P. 1991, MNRAS, 253, 561
Fishman, G. S. 1995, Monte Carlo: Concepts, Algorithms and Applications (Springer)

Harris, A. W., \& Harris, A. W. 1997, Icarus, 126, 450

Hasegawa, S., \& Abe, M. 1999, Lunar Planet. Sci. Conf., 30, 1546

Hasegawa, S. 2001, private communication

Krasinsky, G. A., Pitjeva, E. V., Vasilyev, M. V., \& Yagudina, E. I. 2001, submitted to Icarus

Lupishko, D. F. 1998, Astron. Vestn., 32, 141

Pitjeva, E. V. 2001, Celest. Mech. Dyn. Astr., 80, 249

Small Body Data Archive 2001,

url: http://pdssbn.astro.umd.edu

Standish, E. M. 1998, Jet Propulsion Laboratory IOM 312.F00-048

Standish, E. M. 2000, Jet Propulsion Laboratory IOM 312.F98-107b

Tedesco, E. F., Veeder, G. J., Fowler, J. W., \& Chillemi, J. R. 1992, Phillips Laboratoty Report, PL-TR-92-2049

Williams, J. G. 1984, Icarus, 57, 1

Williams, J. G., \& Standish, E. M. 1989, in Ref. Frames, ed. J. Kovalevsky, I. I. Mueller, \& B. Kolaczek (Kluwer Academic Publishers, Dordrecht) 